\title{
Synthetic lethality with cisplatin by targeting FACT complex in breast cancer
}

\author{
Sarah Swiezy ${ }^{1}$, Rebecca Dirks, $\mathrm{MD}^{2}$, Harikrishna Nakshatri, $\mathrm{PhD}^{3}$ \\ ${ }^{1}$ Indiana University School of Medicine, Department of Surgery and Department \\ of Medical and Molecular Genetics, Indianapolis, IN, ${ }^{2}$ IMPRS Summer Research \\ Program, Indianapolis, IN
}

Background and Hypothesis: Previous work by our lab has shown that FACT (FAcilitates Chromatin Transcription) is upregulated from $\mathrm{P}\left(1^{\circ}\right)$ $\rightarrow \mathrm{I}$ (immortalized) $\rightarrow \mathrm{R}$ (Ras-transformed) cells. The FACT complex is targeted by curaxins, small molecules that "trap" FACT on chromatin, decreasing its ability to destabilize histone tetramers and facilitate DNA replication and transcription. The lab has also observed selective sensitivity to curaxins in the same pattern, $P \rightarrow I \rightarrow R$, suggesting the dependency of Ras-transformed cells on FACT for survival. Therefore, we hypothesized that by impairing the cancer cell's ability to repair DNA, curaxins sensitize breast cancer cells to DNA damage-inducing chemotherapeutics, such as cisplatin. Cisplatin represents the current standard of care for triple negative breast cancer (TNBC), but is associated with exceedingly toxic side effects as well as resistance. Combination therapies may permit cisplatin dose reduction while simultaneously improving response.

Project Methods: We first determined which cancer cell lines (MCF7: ER+/PR+/HER2-; T47D: ER+/PR+/HER2-; and MDA-MB-453: TNBC) would be appropriate for demonstrating synergistic cisplatin + CBL0137 (specific curaxin) cytotoxicity. We then used BrdU assay and Western blot to assess the effects of the drugs individually and in combination on cancer cells.

Results: We observed heterogeneity in sensitivity of cancer cell lines to CBL0137 as well as statistically significant synthetic lethality between cisplatin and CBL0137.

Conclusion and Potential Impact: The knowledge gained by this research is actively being used to develop chemoprevention strategies and to enhance the effectiveness of chemotherapy. Prompted by our results, in vivo preclinical and clinical studies using cisplatin + CBL0137 to treat TNBC are planned. 